\title{
Common upper respiratory tract problems in the elderly-A guide to clinical diagnosis and prudent prescription
}

\section{Geffen MBChB, MCFP(SA)}

To cite this article: $L$ Geffen MBChB, MCFP(SA) (2006) Common upper respiratory tract problems in the elderly-A guide to clinical diagnosis and prudent prescription, South African Family Practice, 48:5, 20-23, DOI: 10.1080/20786204.2006.10873390

To link to this article: http://dx.doi.org/10.1080/20786204.2006.10873390

\section{() 2006 SAAFP. Published by Medpharm.}

\section{曲 Published online: 15 Aug 2014.}

Submit your article to this journal $\llbracket$

Llll Article views: 872 


\section{Common upper respiratory tract problems in the elderly - A guide to clinical diagnosis and prudent prescription}

Geffen $\mathbf{L}, \mathrm{MBChB}, \mathrm{MCFP}(\mathrm{SA})$

Consultant, The Albertina and Walter Sisulu Institute of Ageing in Africa, Faculty of Health Sciences, University of Cape Town

Family Physician, Sea Point

Correspondence: Dr Leon Geffen, E-mail: Igeffen@iafrica.com

\section{Abstract}

Upper respiratory tract infections (URTIs) in the elderly (those aged 65 years and older) are not more common than in younger people, but complications are more common. In uenza and respiratory syncitial virus are the two most common pathogens affecting morbidity and mortality. Hospitalisation, bronchitis, pneumonia and death are all significantly increased in the elderly. In uenza vaccine is recommended for all persons aged 65 and older. Antibiotics have no benefit in the treatment or prevention of complications from in uenza. Otitis media and tonsillitis are uncommon. Sinusitis should be treated with antibiotics. Amoxycillin remains the drug of choice for patients who are not allergic to penicillin. Pneumococcal vaccine is recommended as prophylaxis against pneumococcal pneumonia, which is often seen as a complication of in uenza. Chronic idiopathic cough, anosmia, gastroesophageal re ux disease, asthma and chronic post-nasal drip may all present as upper respiratory tract-like illness. There is no benefit from using common cough mixtures or many other OTC preparations for the management of URTIs, as their side effects are significantly greater in older persons.

SA Fam Pract 2006;48(5): 20-23)

\section{Introduction}

Upper respiratory tract infections (URTIs) in older persons are seen commonly in general practice. Older persons who suffer from an URTI may have decreased daily activity and slower recovery from their illness, may access healthcare services more frequently and may have more severe complications, including death, than their younger cohorts. ${ }^{1}$

Factors that play a role in the severity of disease include immunosenescence, i.e. impaired immune and inflammatory changes that occur with old age. ${ }^{2}$ Older individuals may not respond well to therapy for infections and may also not present with "typical signs" of infection, e.g. fever. Anatomical changes that occur in the upper airways, e.g. reduced ciliary escalator efficiency, cough reflex increase and gastroesophageal reflux disease, may predispose an older person to an increased risk of URTI. Living in close confines, e.g. in a longterm care facility, has been implicated in an increased risk of URTI in older residents. Such populations must be viewed as a specific group of people at high risk of URTI, especially during winter flu epidemics.
Influenza and Respiratory Syncitial Virus (RSV) are the two most common viral URTIs that occur in the elderly. Pneumococcal disease is up to 14 times more common in people aged 70 years and over than in younger individuals. The pneumococcus is a common pathogen in the nasopharynx and is most commonly associated with pneumonia. However, it is also commonly implicated in otitis media and sinusitis.

\section{In uenza}

Influenza is characterised by a sudden onset of fever, muscle aches and pains, malaise, and upper respiratory tract symptoms, including rhinitis, sore throat and non-productive cough. The incubation period typically is one to two days. Some influenza infections are associated with minor symptoms and virological studies have shown that at least $10 \%$ of URTIs in elderly people are due to influenza. ${ }^{3}$ Older persons may not present with the above typical symptoms, and during the influenza season one should suspect influenza if patients present with lassitude, fever and confusion. ${ }^{4}$

Influenza is not more common in older persons than in the general population, although sequelae of influenza are greater. Hospitalisation is up to 20 times more likely in older persons, and especially among those who have concomitant chronic diseases such as cardiac, pulmonary or renal disease. Approximately $25 \%$ of elderly patients will suffer complications due to influenza. Bronchitis is up to 20 times more common and pneumonia up to 10 times more likely to occur. Figure 1 shows the estimated annual mortality associated with influenza and respiratory syncitial virus in the USA for the period 1990-1999. ${ }^{5}$

Figure 1: Estimated annual mortality rates associated with influenza and Respiratory Syncitial Virus (rates per 100000 person-years for seasons 1990-1991 to 1998-1999)

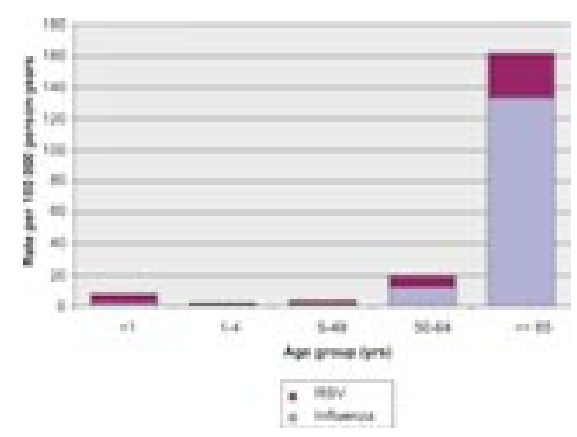


Secondary bacterial pneumonia is the most common and most serious complication post influenza. Bacterial pneumonia is most likely due to Streptococcus pneumoniae. However, Staphylococcal pneumonia accounts for up to a quarter of all post-influenza bacterial pneumonias. With a bacterial pneumonia, there is often an initial improvement in the influenza symptoms, followed five to 10 days later with a relapse of the symptoms, i.e. high fever, productive cough and mucopurulent sputum. A chest X-ray usually shows signs of consolidation.

Unlike bacterial pneumonia, viral pneumonia due to influenza occurs three to five days after the onset of the initial influenza symptoms. Patients typically present with worsening cough, tachypnea, dyspnea and fever. Chest X-rays usually show bilateral patchy infiltrates, although localised infiltrates may occasionally be seen. The disease can progress rapidly over one to four days, resulting in severe respiratory failure and death.

The general practitioner is ideally situated to diagnose influenza in older people, as these practitioners are actively involved in the health of their communities and know when the influenza season begins. The use of viral cultures is the gold standard for diagnosing influenza and typing the strain present in the community. However, the tests are costly and need to be taken within three days of the onset of symptoms, and the results are often not available rapidly enough to initiate antiviral therapy. ${ }^{4}$

Prevention of influenza through the use of an inactivated influe-nza virus vaccine annually is recommended. An influenza vaccine is recommended for all persons aged 65 years and older and all residents of long-term care facilities. Vaccination is also recommended for persons who can transmit influenza to elderly patients, such as healthcare workers, community workers who provide services to the elderly and persons in frequent contact with older persons, e.g. family members.

The influenza vaccine is not universally effective in producing an antibody response and only 40 to $60 \%$ of older persons develop an effective immune response within 10 to 14 days of receiving the vaccine. However, the primary goal of the influenza vaccine is not to prevent influenza epidemics, but rather to reduce the serious sequelae of the disease. Prevaccination titres of antibodies to influenza and the number of previous influenza vaccinations have been shown to influence post-vaccination titres.

Vaccination against influenza has been shown to significantly reduce the risk of hospitalisation for heart disease, cerebrovascular disease and pneumonia, which all cause mortality during an influenza season. The risks associated with the influenza vaccine are negligible and its use is only contraindicated in people with an egg allergy. Regular, repeated vaccinations are more effective and persons who have had annual vaccinations for the prior four years are more likely to develop immunity. Studies have shown that the most common reason why most people do not receive vaccination is a lack of information from their GP, a fear of side effects and concern about vaccine efficacy. It is thus incumbent upon a GP to offer an influenza vaccine to all eligible elderly patients.

Antiviral drugs, such as amantadine and zanamavir, are available for the treatment of influenza. These drugs need to be used within 48 hours of the start of symptoms. Amantadine has been shown to be effective in nursing homes as prophylaxis during an influenza outbreak. The drugs are not a substitute for the influenza vaccine and should be used as an adjunct to the vaccine. Amantadine is only effective against influenza and has been shown to reduce disease severity. However, due to neurological side effects and the rapid emergence of resistant strains, its use has been limited. The recommended dose of amantadine is $100 \mathrm{mg}$ daily. It is excreted via the kidneys and thus should be used with caution in patients with renal impairment. When an outbreak of influenza occurs in a nursing home, amantadine should be used for a minimum of two weeks.

Zanamavir is a neuraminidase inhibitor that has been shown to reduce the symptoms of influenza by 0.9 days and evidence for its effectiveness in treating high-risk groups is limited. ${ }^{6}$

\section{Respiratory Syncitial Virus}

Respiratory Syncitial Virus (RSV), together with rhinovirus and influenza virus, is among the three most common viral pathogens identified in hospitalised patients in the winter. ${ }^{4,7}$ It is a paromyxovirus and an important cause of lower respiratory tract infections in elderly patients, particularly those living in long-term care facilities and in households with children.

Clinical symptoms are not unlike influenza, but may range from those of a mild cold to severe respiratory distress. Fever occurs in only 50\% of patients, and the patients are more commonly found to have signs of lower respiratory tract infection, i.e. wheezes and crackles. RSV infection may be diagnosed using laboratory techniques such as viral cultures from the nasopharynx, RSV-specific IgM, or a four-fold increase in IgG titres between acute and convalescent phase serum samples. In elderly patients there is a reduced sensitivity to these tests, and due to their cost they are not recommended routinely. Rapid tests are being developed and may become more accessible within general practice in the near future.

The treatment of RSV in the elderly is mainly supportive, i.e. adequate hydration, oxygen and bronchodilators. Aerosolised ribavirin is approved in the USA, but is not available in South Africa. Evidence as to its efficacy in older persons is lacking and in the USA it is seldom used.

\section{Sinusitis, otitis media and tonsillitis}

There is a lack of evidence on the management of sinusitis, otitis media and sinusitis in elderly people. The GP must therefore be guided by the 
recommendations of clinical guidelines for all adults.

Streptococcal pharyngotonsillitis is uncommon in adults older than 45. The presence of a temperature $>38{ }^{\circ} \mathrm{C}$, tonsillar exudates, anterior cervical and an acute onset of sore throat point towards bacterial pharyngotonsillitis. The presence of rhinorhea, cough, diarrhoea and conjunctivitis mitigate against a bacterial aetiology. South African guidelines for management recommend the use of penicillin VK 500 $\mathrm{mg}$ bid for 10 days, or benzathine penicillin $1.2 \mathrm{mu}$ IM. For penicillinallergic individuals, an erythromycinbased antibiotic is recommended.

Otitis media is relatively uncommon in older persons, although if a bacterial infection is suspected, the most likely organism is a streptococcus. Current guidelines suggest the use of amoxicillin 500$1000 \mathrm{mg}$ three times a day for five days. In penicillin-allergic persons, the recommended alternative remains an erythromycin-based antibiotic for five to seven days, except in the case of clarithromycin, which can be used for three days.

Sinusitis should be suspected in individuals who have an upper respiratory tract infection that is not improving after 10 days or worsens after five to seven days. It is accompanied by some of the following symptoms: fever, facial tenderness over the sinuses, dental tenderness, nasal discharge, anosmia and nasal congestion. The most likely bacterial pathogen is the streptococcus. Amoxycillin 500$1000 \mathrm{mg}$ three times a day for 10 days is recommended. In penicillinallergic individuals, a macrolide or quinolone-based antibiotic is recommended for 10 days. Should a patient not respond within 72 hours, have periorbital swelling, have any signs of CNS involvement, e.g. meningism and/or an altered level of consciousness or focal neurological signs or severe systemic illness, the patient should be referred to a specialist for further management.

Polyvalent pneumococcal vaccine (Pneumovax) causes a B-cell response. The response is ad- vantageous in older people, as immunosenescence affects mostly T-cell immunity. It is thus advisable to vaccinate all people over the age of 65 with a pneumococcal vaccine. The vaccine may be re-administered every five years. Some studies have shown a prevention rate of pneumonia of as high as 60-95\% in immunocompetent older and highrisk patients. ${ }^{9}$

\section{Pitfalls in management}

Most upper respiratory tract infections are self-limiting and the wise GP knows that, in many instances, watchful waiting together with the body's own defence system will deal with the majority of problems. Recently, a number of conditions that may mimic URTIs have been described. The concern with not having a correct diagnosis is that people will make use of over-the-counter preparations for the treatment of their conditions, thereby leading to unnecessary expense and, most importantly, unwarranted side effects from the myriad of less than efficacious drugs available from pharmacies and supermarkets.

Olfactory impairment exists in about $4.6 \%$ of people over the age of 75 and the prevalence increases with age. It is important to be able to distinguish that a loss of smell in older persons is a physiological change associated with ageing and is not due to an infection. ${ }^{10}$

Chronic cough is defined as lasting longer than eight weeks. The most common causes of chronic cough are asthma, gastroesophageal reflux disease and post-nasal drip. No cause is found in a small group of patients, even after extensive investigation. These patients are labelled as having chronic idiopathic cough $(\mathrm{ClC})$. Classically, patients will have had an initial minor URTI that triggers the cough, yet, despite the infection subsiding, the cough persists for months or years. These patients have often tried a number of empiric therapies, all of which are ineffective. The patients tend to have very sensitive cough reflexes and cough is triggered by eating crumbly foods, such as bread and biscuits, and exposure to strong smells from perfume. The mechanism of $\mathrm{ClC}$ is unknown, but it is thought to be due to hyperalgesia with a reduction in sensory nerve stimulation to pain. ${ }^{11}$

\section{Conclusion}

Upper respiratory tract infections are mostly self-limiting, although complications resulting from influenza and other viral aetiologies can have dire consequences in older persons. The general practitioner has been shown to be an effective agent for getting patients to accept the influenza vaccine. GPs must be prudent in advising treatments with little or no efficacy, such as antibiotics and over-the-counter cough and "cold" remedies, in the hope that these will lessen the patient's illness. Most evidence suggests that most URTIs will get better and the harm done by unproven therapies can be far greater than the good done. The GP is the person who is placed best to educate and inform patients about the best practice in managing URTIs.

\section{See CPD Questionnaire, page 50}

\section{(P) This article has been peer reviewed}

\section{References}

1. Meydani SN, Leka LS, Fine BC, et al. Vitamin E and respiratory tract infections in elderly nursing home residents. JAMA 2004;292:828-36.

2. Gravenstein S, Fillit HM, Ershler WB. Clinical immunology of aging. In: Tallis RC, Fillit HM, eds. Brocklehurst's textbook of Geriatric Medicine and Brocklehurst's textbook of Geriatric Medicine and
Gerontology. $6^{\text {th }}$ edition. Churchill Livingston; 2003. p. 117-8.

3. Stott DJ, Carman WF, Elder AG. Influenza in old age Age and Ageing 2001;30:361-3.

4. Bader MS, McKinsey DS. Viral infections in the elderly: the challenges of managing herpes zoster influenza and RSV. Postgrad Med 2005;118(5):45

5. Thompson WW, Shay DK, Weintraub E, et al. Mortality associated with influenza and respiratory syncytial virus in the United States. JAMA 2003:289: 179-86.

6. Cooper NJ, Sutton AJ, Abrams KR, et al. Effectiveness of neuraminidase inhibitors in treatment and prevention of influenza $A$ and $B$ systematic review and meta-analyses of randomised control trials. BMJ 2003;326:1235-40.

7. Falsey AR, Hennessey RN, Formica MA, et al. Falsey AR, Hennessey RN, Formica MA, et al.
Respiratory Syncitial Virus Infection in elderly and high risk adults. N Engl J Med 2005;352:1749-59.

8. Brink AJ, Cotton MF, Feldman C, Geffen L, et al Working Group of the Infectious Diseases Society of South Africa. Guideline for the management of upper respiratory tract infections. S Afr Med J 2004;94:47583.

9. Yamaya M, Yanai M, Ohrui T, et al. Interventions to prevent pneumonia among older adults. JAGS 2001:49:85-91.

10. Murphy C, Schubert CR, Cruickshanks KJ, et al. Prevalence of olfactory impairment in older adults. JAMA 2002.288.2307-12

11. Haque RA, Usmani OS, Barnes PJ. Chronic idiopathic cough: a discrete clinical entity? Chest 2005;127:1710-3 